\title{
Aminoácidos protegidos na ração de borregas sobre o consumo, desempenho e comportamento ingestivo
}

\section{Carolina Moreira Araújo $^{1 *}$, Karla Alves Oliveira ${ }^{2}$, Gilberto de Lima Macedo Junior ${ }^{3}$, Adriana Lima Silva ${ }^{4}$, Débora Adriana de Paula Silva ${ }^{5}$, Marco Tulio Santos Siqueira ${ }^{6}$}

\begin{abstract}
Resumo
Objetivou-se avaliar o consumo, desempenho e comportamento ingestivo de borregas suplementadas com lisina e metionina protegidas da degradação ruminal. Utilizou-se 20 borregas com peso e idade média de $38 \pm 5 \mathrm{~kg}$ e seis meses, respectivamente, divididas em quatro baias coletivas, durante 105 dias de período experimental (sendo 15 dias de adaptação e 90 de coleta de dados). As dietas eram compostas por silagem de milho e concentrado: com inclusão de $4 \%$ de MicroPEARLS ${ }^{\circledR}$, sendo este composto por lisina e metionina protegidos, caracterizando os tratamentos:sem a inclusão do produto (Tratamento controle) e com a inclusão (Tratamento Lis + Met), no concentrado. O consumo de matéria seca (CMS) foi avaliado diariamente, as medidas biométricas, escore de condição corporal (ECC) e peso foram obtidos a cada 15 dias. O delineamento experimental foi inteiramente ao acaso com dez repetições (animais) por tratamento para as variáveis biométricas e comportamento ingestivo. O delineamento experimental foi inteiramente ao acaso, com medidas repetidas ao tempo com dez repetições (animais) por tratamento para as variáveis biométricas e comportamento ingestivo. Na parcela ficou a inclusão ou não do aminoácido e como medida repetida ao tempo o período (sendo este avaliado por estudo de regressão a $5 \%$ de probabilidade por se tratar de variáveis quantitativas). O consumo de matéria seca em função dos tratamentos foi comparado utilizando-se estatística descritiva. As medidas biométricas não foram influenciadas pelos tratamentos, e aumentaram de forma linear no período avaliado. Houve interação entre tratamento e período para altura de posterior e comprimento corporal. O ganho médio diário e comportamento ingestivo não foram influenciados. A inclusão de aminoácidos (lisina e metionina) protegidos em até 4\% não altera o CMS, ganho de peso, desenvolvimento corporal e o comportamento ingestivo de borregas.
\end{abstract}

Palavras-chave: Lisina. Medidas biométricas. Metionina. Ovis aries.

\section{Protected amino acids in control of consumption, performance and ingested behavior}

\author{
Abstract \\ ${ }^{1}$ Universidade Federal de Uberlândia, Uberlândia, MG. Brasil. \\ https://orcid.org/0000-0003-4648-4971 \\ ${ }^{2}$ Universidade Estadual Paulista, Jaboticabal, SP. Brasil. \\ http://orcid.org/0000-0002-7792-2615 \\ ${ }^{3}$ Universidade Federal de Uberlândia, Uberlândia, MG. Brasil. \\ http://orcid.org/0000-0001-5781-7917 \\ ${ }^{4}$ Universidade Federal de Uberlândia, Uberlândia, MG. Brasil. \\ http://orcid.org/0000-0002-9153-3317 \\ ${ }^{5}$ Universidade Federal de Uberlândia, Uberlândia, MG. Brasil. \\ http://orcid.org/0000-0003-3052-0544 \\ ${ }^{6}$ Universidade Federal de Uberlândia, Uberlândia, MG. Brasil. \\ http://orcid.org/0000-0002-2098-8568 \\ *Autor para correspondência: carolina.am@hotmail.com
}

The objective of this study was to evaluate the intake, performance and ingestive behavior of lambs supplemented with lysine and methionine protected from ruminal degradation. Twenty lambs with average weight and age of 38 $\pm 5 \mathrm{~kg}$ and six months, respectively, were divided into four collective stalls during 105 days of experimental period (15 days of adaptation and 90 days of data collection). The diets were composed of corn silage and concentrate: with 4\% MicroPEARLS ${ }^{\circledR}$ inclusion, which is protected lysine and methionine, characterizing the treatments: without the inclusion of the product (Control treatment) and with the inclusion (Lis + Met treatment) in the concentrate. Dry matter intake (DMI) was evaluated daily, biometric measurements, body condition score (BCS) and weight were

Recebido para publicação em 02 de novembro de 2019. Aceito para publicação em 11 de dezembro de 2019 e-ISSN: 2447-6218 / ISSN: 2447-6218 / (C) 2009, Universidade Federal de Minas Gerais, Todos os direitos reservados. 
obtained every 15 days. The experimental design was completely randomized with ten replications (animals) per treatment for biometric variables and ingestive behavior. The experimental design was completely randomized, with measurements repeated over time with ten replications (animals) per treatment for biometric variables and ingestive behavior. In the plot was the inclusion or not of the amino acid and as a measure repeated over time the period (this being evaluated by regression study at $5 \%$ probability because they are quantitative variables). Dry matter intake as a function of treatments was compared using descriptive statistics. Biometric measurements were not influenced by treatments and increased linearly over the period evaluated. There was interaction between treatment and period for posterior height and body length. Average daily gain and ingestive behavior were not influenced. Inclusion of protected amino acids (lysine and methionine) by up to $4 \%$ does not alter DMI, weight gain, body development and ingestive behavior of lambs.

Keywords: Lysine. Biometric measurements. Methionine. Ovis aries.

\section{Introdução}

A qualidade da proteína absorvida pelos ruminantes é dependente do perfil de aminoácidos que chega ao intestino delgado, e não dos aminoácidos ingeridos, tendo em vista que aqueles que são fornecidos, via dieta, são intensamente metabolizados no ambiente ruminal e convertidos em proteína microbiana (Alves et al., 2010).

A proteína microbiana juntamente com aquela que escapada degradação ruminal e chegam ao intestino delgado serão utilizadas para atender as exigências de proteína metabolizável, sendo este termo aplicado ao total de aminoácidos absorvidos pelo animal (Van Soest, 1994). Neste sentido, atender as exigências de proteína metabolizável dos ruminantes torna-se um processo complexo, tendo em vista que o perfil de aminoácidos que chega ao intestino delgado nem sempre é aquele requerido pelos animais.

Além disso, os alimentos utilizados na alimentação de animais ruminantes apresentam deficiência em lisina e metionina, sendo estes os aminoácidos mais limitantes para sua produção (Stieven et al. 2011).

Segundo Macedo Junior et al., (2019) quando a proteína metabolizável possui alto valor biológico, ou seja, possui perfil de aminoácidos essenciais adequado, o teor de proteína bruta da dieta pode ser reduzido, a eficiência de utilização da proteína metabolizável é melhorada, consequentemente, a exceção de ureia e demais compostos nitrogenados é reduzida, maximizando o desempenho dos animais.

Lisina e metionina são considerados importantes para o crescimento microbiano, fermentação ruminal, produção de leite e crescimento de lã (NRC, 2007). Os requisitos de lisina e metionina digestíveis que permite maximizar o uso da proteína metabolizável na síntese de proteína é de 2,4 e 7,2\% na proteína metabolizável, respectivamente, resultando em uma relação de 3:1. Esta relação foi obtida levando-se em consideração as proporções destes dois aminoácidos presentes no leite e tecido muscular (NRC, 2007).

O fornecimento de lisina e metionina encapsulados podem reduzir os gastos energéticos no metabolismo animal, uma vez que há um maior aporte de aminoácidos essenciais para o intestino delgado e menor excreção de compostos nitrogenados para o ambiente.

Com o crescente aumento no consumo de produtos de origem animal torna-se fundamental a busca por uma alimentação mais eficiente, que proporcione maiores ganhos de peso. Por isso, a avaliação do desempenho animal através do estudo da biometria corporal e comportamento ingestivo são parâmetros que auxiliam na tomada de decisões. Além disso, ainda são escassos os trabalhos avaliando o efeito da inclusão de aminoácidos na dieta de borregas.

Neste sentido, objetivou-se avaliar a inclusão de lisina e metionina sobre o consumo, desempenho e comportamento ingestivo de borregas.

\section{Material e métodos}

O experimento foi conduzido na Fazenda Experimental Capim Branco da Universidade Federal de Uberlândia, no setor de ovinos e caprinos, durante os meses de junho a agosto de 2016, com 105 dias de duração, sendo 15 dias para adaptação dos animais e 90 dias para coleta de dados.O projeto foi aprovado pelo Comitê de ética em experimentação animal da Universidade Federal de Uberlândia, sob protocolo número 149/16

Foram utilizadas vinte borregas mestiças $3 / 4$ Dorper $\mathrm{x} 1 / 4$ Santa Inês, não gestantes, com aproximadamente cinco meses de idade, peso corporal médio de $38 \pm 5 \mathrm{~kg}$ e escore de condição corporal médio de 2,77 , as quais foram distribuídas aleatoriamente em quatro baias de piso ripado, sendo cinco animais para cada baia, sendo duas baias para cada tratamento, totalizando dez repetições (animais) por tratamento. O número de baias foi utilizado como repetição para a avaliação do consumo de matéria seca (sendo então, duas repetições), e o número de animais foi utilizado como repetição para as demais avaliações. As baias continham saleiro, bebedouro e cocho coletivo, com separação tipo canzil com capacidade para alocar seis animais por baia.

Antes do período experimental os animais eram mantidos em quatro baias coletivas com cinco animais cada, em grupos diferentes dos formados no presente 
Aminoácidos protegidos na ração de borregas sobre o consumo, desempenho e comportamento ingestivo

estudo. Os animais eram identificados com brincos com numeração diferenciada, permitindo a identificação de cada animal. As baias eram de piso ripado, dispostas de bebedouros, comedouro e saleiros. A alimentação era composta por volumoso e concentrado extrusados, na relação 50V:50C. Para o início do experimento, as borregas foram mantidas em jejum alimentar por 12 horas e, posteriormente, pesadas com o auxílio de balança manual da marca João Trivelato ${ }^{\circledR}$ (Rolândia/PR)com capacidade para $300 \mathrm{~kg}$ e precisão de 20 gramas, e vermifugadas com monepantel, via oral, (1mL/ 10kg de peso corporal).
Os animais receberam diferentes dietas de acordo com os seguintes tratamentos: concentrado com inclusão de $4 \%$ do produto MicroPEARLS LM ${ }^{\circledR}$, sendo este um suplemento de aminoácidos (lisina e metionina; Tratamento Lis + Met) encapsulados e concentrado sem inclusão do produto (Controle). O produto foi adicionado ao concentrado no momento de sua fabricação, na fazenda experimental, promovendo homogeneidade na mistura. As dietas eram compostas por silagem de milho e concentrado (Tabela 1) e balanceadas para ganhos de 200g/ dia segundo NRC (2007), na relação 30V:70C.

Tabela 1 - Composição centesimal dos concentrados nos tratamentos e composição bromatológica do concentrado, silagem de milho, MicroPEARLS e ração total.

\begin{tabular}{|c|c|c|c|c|}
\hline Ingredientes & \multicolumn{2}{|c|}{ Controle (\%) } & \multicolumn{2}{|c|}{ Lis + Met (\%) } \\
\hline Milho moído & \multicolumn{2}{|c|}{60,50} & \multicolumn{2}{|c|}{60,00} \\
\hline Farelo de soja & \multicolumn{2}{|c|}{36,00} & \multicolumn{2}{|c|}{32,50} \\
\hline Ureia & \multicolumn{2}{|c|}{1,00} & \multicolumn{2}{|c|}{1,00} \\
\hline MicroPEARLS & \multicolumn{2}{|c|}{0,00} & \multicolumn{2}{|c|}{4,00} \\
\hline Sal mineral ${ }^{1}$ & \multicolumn{2}{|c|}{2,50} & \multicolumn{2}{|c|}{2,50} \\
\hline Nutrientes & Concentrado $^{2}(\%)$ & Silagem de milho $^{2}$ & MicroPEARLS $^{\circledR 3}$ & Ração total $^{2}$ \\
\hline MS & 90,20 & 32,20 & 99,00 & 43,56 \\
\hline PB & 24,90 & 6,30 & 43,75 & 19,39 \\
\hline FDN & 24,80 & 54,60 & - & 33,74 \\
\hline FDA & 7,00 & 32,60 & - & 14,68 \\
\hline NDT & 80,94 & 62,63 & & 65,05 \\
\hline
\end{tabular}

${ }^{1}$ Calcário calcítico, cloreto de sódio, enxofre ventilado, fosfato bicálcio, óxido de magnésio, melaço, transquelato de cobre, sulfato de cobalto, sulfato de manganês, selenito de sódio, iodato de cálcio, tranquelato de zinco, óxido de zinco, tranquelato de selênio. ${ }^{2}$ Informações obtidas no laboratório de nutrição animal da UFU. ${ }^{3}$ Informações cedidas pelo fabricante; Produto composto por $75 \%$ lisina e $25 \%$ metionina. MS - matéria seca; PB proteína bruta; NDT - nitrogênio digestível total; FDN - fibra em detergente neutro; FDA - fibra em detergente ácido; EE - extrato etéreo. NDT = $87,84-(0,779$ x \%FDA) (Rodrigues, 2010)

A oferta da ração foi realizada duas vezes ao dia (8 e 16h), sendo ofertada a mesma quantidade nos dois horários. Todos os dias, as quantidades ofertadas e as sobras de alimento foram pesados com o auxílio de balança eletrônica com precisão de cinco gramas para acompanhamento do consumo pelos animais. As rações foram fornecidas de forma que houvesse sobra de $10 \%$ do total fornecido. As sobras de alimento foram colhidas e armazenadas em sacos plásticos, devidamente identificados, e mantidas em congelador a $-10^{\circ} \mathrm{C}$. Após o período de coleta (a cada sete dias) as amostras eram homogeneizadas individualmente e uma alíquota representativa era separada para realização das análises bromatológicas, conforme proposto por Silva e Queiroz (2002).

Posteriormente, foi feita a primeira secagem das amostras de sobras em estufa de circulação forçada de ar, a $55^{\circ} \mathrm{C}$ por 72 horas, até obter peso constante. Feito isto, as amostras foram moídas, em moinho de facas do tipo Willey dotados com peneiras com crivos de diâmetro de 1 $\mathrm{mm}$. Logo após, as amostras foram levadas ao laboratório onde foi realizada a $2^{\mathrm{a}}$ matéria seca, permitindo calcular o consumo de matéria seca (CMS) pelos animais através da fórmula: $\mathrm{CMS}=$ (ofertado de alimento $\mathrm{x} \%$ matéria seca do ofertado) - (sobras de alimento $\mathrm{x} \%$ matéria seca das sobras). Como o alimento ofertado e as sobras eram relativos à baia, o CMS também foi calculado em relação às baias, sendo assim, os resultados referem-se ao consumo dos cinco animais de cada baia, assemelhando-se ao que ocorre na prática nas propriedades.

As mensurações para a determinação das medidas biométricas foram realizadas a cada 15 dias, totalizando sete avaliações, no período da manhã, antes da primeira oferta de alimento. As mensurações foram realizadas com os animais com as quatro patas apoiadas no chão, em local plano, com auxílio de fita métrica e bastões de madeiras adaptados para tal função, de acordo com a metodologia proposta por Cezar e Souza (2007). Foram mensurados: 
- $\quad$ Altura do posterior (AP): distância entre o ponto dorsal da tuberosidade coxal e o ponto mais distal do membro posterior, tomada na posição vertical e paralelamente a face lateral do membro;

- Altura do anterior (AA): distância vertical entre o ponto mais alto (no sentido da escápula) e o solo;

- Circunferência torácica (CT): é obtida contornando-se a caixa torácica, passando pelo dorso, cilhadouro e costado;

- Circunferência do barril (CB): obtida contornando-se a região do vazio;

- $\quad$ Largura de peito (LP): distância entre as faces laterais das articulações escápulo-umerais do lado direito e esquerdo;

- Comprimento corporal (CC):distância regional do corpo que vai do ponto de encontro entre o pescoço e a cernelha até o ponto de encontro entre a garupa e a cauda.

- $\quad$ Escore de condição corporal (ECC):obtido palpando-se a região lombar dos animais e atribuindo-se notas de 1 a 5 (Kenyon et al., 2014).

Para a mensuração do comportamento ingestivo, os animais foram submetidos à observação visual por pessoas treinadas, em sistema de revezamento, dispostas de maneira a não incomodar os animais, por 24 horas. No período noturno, o ambiente recebeu iluminação artificial, e as luzes foram mantidas acessas cinco dias antes da avaliação para promover a adaptação dos animais. Foram verificados, a cada cinco minutos, se os animais estavam realizando ingestão do alimento e água (ING), ruminação (RUM) ou ócio (ÓCIO), de acordo com a metodologia proposta por Fischer et al., (1998). Os cálculos das atividades foram feitos em minutos por dia, admitindo que, nos cinco minutos subsequentes a cada observação, o animal permaneceu na mesma atividade. Já o tempo total gasto em mastigação (MAST) foi determinado somando-se os tempos gastos em ingestão (ING) e ruminação (RUM). Estas avaliações ocorreram nos dias 1, 45 e 90 dias de experimento, objetivando verificar possíveis alterações no comporto ingetivo dos animais ao longo de todo o período experimental, além de auxiliar na avaliação da qualidade da dieta.

O delineamento experimental foi inteiramente ao acaso, com medidas repetidas ao tempo com dez repetições (animais) por tratamento para as variáveis biométricas e comportamento ingestivo. Na parcela ficou a inclusão ou não do aminoácido e como medida repetida ao tempo o período (sendo este avaliado por estudo de regressão a $5 \%$ de probabilidade por se tratar de variáveis quantitativas).O consumo de matéria seca em função dos tratamentos foi comparado utilizando-se estatística descritiva (por não haver repetição suficiente, tinha apenas duas baias como repetição) e o escore de condição corporal por estatística não paramétrica (Kruskal e Wallis, 1952), por se tratar de uma variável de avaliação subjetiva.As variáveis normais e com variâncias homogêneas foram submetidas análise de regressão linear observando-se a significância dos efeitos linear, quadrático e de desvio da linearidade, sendo a significância utilizada para tomada de decisão $5 \%$ de probabilidade de erro tipo I pelo teste F. Os dados foram submetidos aos testes de normalidade (Shapiro e Wilk, 1965) e homocedasticidade (Cochran, 1941).

$$
y_{i j k}=\mu+\alpha_{i}+e_{i(j)}+\beta_{k}+\alpha \beta_{i k}+\varepsilon_{i j k}
$$

em que com: $\mathrm{i}=1,2, \ldots$, I níveis da parcela; $\mathrm{j}=1,2, \ldots$, $\mathrm{J}$ repetiçoes; $\mathrm{k}=1,2, \ldots, \mathrm{K}$ níveis de medidas repetidas no tempo;

yijk é o valor observado na medida repetida no tempo correspondente ao k-ésimonivel, dentro do i-ésimo nível de parcela, no j-ésimarepetiçao;

$\mu$ é a média geral;

$\alpha_{i}$ é o efeito do i-ésimo nível da parcela;

$e_{i(j)}$ é o erro associado do i-ésimo nível de parcela, no j-ésima repetição

$\beta_{k}$ é o efeito do k-ésimo nível da medida repetida no tempo;

$\alpha \beta_{i k}$ é o efeito de interação entre o i-ésimo nível da parcela e o k-ésimo nível de medida repetida no tempo;

$\varepsilon_{\text {ijk }}$ é o erro aleatório atribuído a observação yijk , considerado como o componente do resíduo.

\section{Resultados e discussão}

O consumo de matéria seca (CMS) ( $\mathrm{kg} / \mathrm{dia})$ foi avaliado de forma descritiva, pois não havia repetições de baias suficientes para comparar os tratamentos. De acordo com o NRC (2007), o CMS recomendado para esta categoria animal é de $1,57 \mathrm{~kg} / \mathrm{dia}$. Os animais alimentados com o tratamento controle consumiram em média $1,42 \mathrm{~kg} /$ animal/dia (10,56\% abaixo do recomendado), enquanto aqueles que receberam o tratamento Lis + Met consumiram 1,50 kg/animal/dia (4,6\% abaixo do recomendado). Estes valores são obtidos dividindo o CMS referente a cada baia pelo número de animais, que era cinco. Neste sentido, podemos concluir que os animais suplementados com aminoácidos encapsulados consumiram numericamente $5,96 \%$ a mais que aqueles que não receberam o produto (controle).Entretanto, vale ressaltar que ambos os tratamentos proporcionaram um CMS bem próximo ao recomendado pela literatura, indicando o atendimento às exigências nutricionais desta categoria. Araújo et al., (2019) testaram níveis de inclusão de lisina e metionina protegidos da degradação ruminal (de 0 a 32 g/animal/dia) na ração de borregas mestiças e observaram aumento no CMS a medida em que aumentaram os níveis de inclusão de lisina e metionina protegidos na ração. Segundo estes autores, o aumento 
na inclusão de aminoácidos protegidos associados aos ingredientes da ração (farelo de soja, farelo de milho, ureia), proporcionaram melhor atendimento às exigências de proteína metabolizável e maior produção de proteína microbiana no rúmen, respectivamente. Este equilíbrio entre as fontes de proteína pode ter resultado nos incrementos numéricos observados para CMS (Tabela 2).

Tabela 2 - Consumo de matéria seca (CMS) em kg/dia referente a cada baia em função dos tratamentos Controle e Lis + Met de borregas mestiças.

\begin{tabular}{cc}
\hline Tratamento & CMS \\
\hline Controle & 7,11 \\
Lis + Met & 7,49 \\
\hline
\end{tabular}

No período avaliado observou-se efeito quadrático no CMS (kd/dia) apresentando queda a partir da oitava semana (Figura1).O aumento no CMS na fase inicial do experimento está relacionado ao aumento nas exigências nutricionais dos animais, por estarem em fase de crescimento, momento este onde há priorização para o crescimento ósseo e músculos (Gois et al., 2018). Já a diminuição no consumo pode estar relacionada com o fato dos animais estarem entrando na fase de puberdade a partir da $8^{\circ}$ semana de experimento, ou seja, em média sete meses de idade, sendo este momento caracterizado por uma alteração na composição do crescimento do animal, priorizando a deposição de gordura, e não mais de ossos e músculos, como mencionado anteriormente. Segundo Gois et al., (2018), após o nascimento, o crescimento apresenta um comportamento lento na fase inicial, aumentando rapidamente logo a seguir, e desacelerando a partir da puberdade. Monteiro et al., (2010), relataram que a puberdade em fêmeas ovinas ocorre entre 4 e 14 meses de idade, sendo influenciada pela genética, manejo alimentar, entre outros. Sendo assim, o CMS observado no presente estudo está diretamente relacionado a estes fatores, uma vez que sua queda ocorreu quando os animais apresentavam aproximadamente sete meses de idade.

Figura 1 - Consumo de matéria seca em função do período avaliado, em semanas.

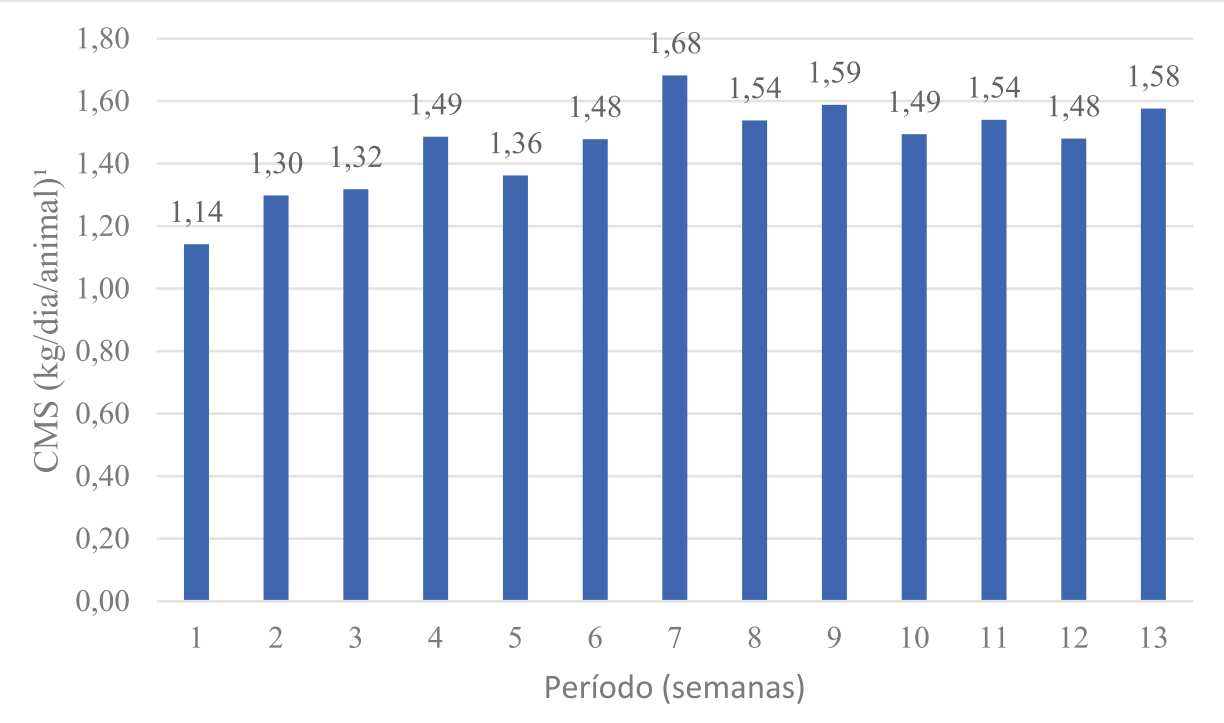

${ }^{1} Y=5,383934+0,524512 x-0,027786 x^{2} R^{2}=77,72 \% ; P=0,0003 ;$ Coeficiente de variação $=11,00 \%$.

Ao final do experimento mesmo com a redução no CMS os animais estavam consumindo $7,88 \mathrm{~kg} / \mathrm{dia} /$ baia, ou seja,em média $1,58 \mathrm{~kg} / \mathrm{dia} / \mathrm{animal}$, exatamente o proposto pelo NRC (2007). Sendo assim, podemos concluir que as dietas foram eficientes em atender a demanda nutricional dos animais, como pode ser visto pelo ganho de peso linear dos animais na Tabela 3.Os parâmetros avaliados na análise de biometria corporal não foram influenciados $(\mathrm{p}<0,05)$ pelos tratamentos e apresentaram equação linear positiva para todas as características avaliadas nos diferentes períodos. Sendo assim, pode-se inferir que ambos os tratamentos se mostraram eficazes para promover desenvolvimento corporal adequado dos animais, corroborando com os dados visto no Figura 1.
Pode-se observar que a partir dos 60 dias de experimento alguns parâmetros avaliados começaram a apresentar estabilização, como alturas do anterior e posterior e largura de peito. Estes resultados podem estar relacionados com o avanço na idade dos animais, vindo a atingir a puberdade, momento este onde há uma desaceleração no crescimento dos animais (Gois et al., 2018), como já mencionado anteriormente. A maturidade da carcaça de ovinos pode ser determinada, entre outros fatores, através da observação da estrutura óssea, uma vez atingido os tamanhos ideais para raça e sexo há uma estabilização no crescimento ósseo, podendo então haver variações apenas em variáveis relacionadas ao peso dos animais. Isso explica a redução no consumo de matéria seca pelos animais. Costa Júnior et al., (2006), encontrou valores semelhantes ao do presente estudo, sendo 
Araújo, C. M. et al.

de 68,2 $\pm 5,1$ e 75,9 $\pm 8,3 \mathrm{~cm}$ respectivamente para AA e CT, para ovinos Santa Inês, com idade caracterizada pela presença de "dentes de leite", indicando que eram animais jovens, mantidos em sistemas de médio nível tecnológico (criação em pastagem nativa e cultivada, com suplementação alimentar na entressafra).

Tabela 3 - Altura do anterior (AA), circunferência torácica (CT), circunferência do barril (CB), largura de peito (LP), escore de condição corporal (ECC) e peso de borregas mestiças em função dos tratamentos e períodos avaliados e interação para altura do posterior (AP) e comprimento corporal (CC).

\begin{tabular}{ccccccc}
\hline Tratamento & AA & CT & CB & LP & ECC & Peso \\
\hline Controle & 63,42 & 84,22 & 95,38 & 21,24 & 3,29 & 47,54 \\
Lis + Met & 62,18 & 83,72 & 96,08 & 20,75 & 3,22 & 46,82 \\
\hline Período & AA $^{1}$ & CT $^{2}$ & CB $^{3}$ & LP $^{4}$ & ECC $^{5}$ & Peso $^{6}$ \\
\hline 0 & 58,30 & 77,95 & 88,35 & 18,75 & 2,77 C & 37,11 \\
15 & 58,65 & 78,70 & 89,45 & 18,15 & 3,12 B & 40,61 \\
30 & 62,75 & 81,25 & 91,65 & 19,95 & 3,10 B & 43,21 \\
45 & 63,65 & 83,75 & 94,45 & 21,45 & $3,37 \mathrm{AB}$ & 47,02 \\
60 & 64,85 & 86,45 & 99,05 & 22,10 & $3,42 \mathrm{~A}$ & 51,24 \\
75 & 65,60 & 88,45 & 101,90 & 23,05 & $3,47 \mathrm{~A}$ & 54,00 \\
90 & 65,85 & 91,30 & 105,30 & 23,55 & $3,52 \mathrm{~A}$ & 57,07 \\
\hline MG & 62,80 & 83,97 & 95,73 & 21,00 & 3,25 & 47,18 \\
CV & 6,77 & 2,21 & 2,57 & 4,71 & - & 3,78 \\
\hline
\end{tabular}

Interação entre tratamento $x$ período

\begin{tabular}{cccrr}
\hline \multirow{2}{*}{ Período } & \multicolumn{3}{c}{ AP } \\
\cline { 2 - 5 } 0 & Lis + Met $^{7}$ & Controle $^{8}$ & ${\text { Lis }+ \text { Met }^{9}}^{\text {CC }}$ & Controle $^{10}$ \\
15 & 59,50 & 59,70 & 66,30 & 63,10 \\
30 & 61,40 & 62,60 & 66,00 & 64,70 \\
45 & 62,80 & 64,30 & 68,30 & 67,10 \\
60 & 64,80 & 65,30 & 70,10 & 69,60 \\
75 & 65,90 & 66,50 & 71,10 & 72,10 \\
90 & 66,70 & 66,60 & 72,80 & 72,70 \\
MG & 67,00 & 66,80 & 72,90 & \\
CV & \multicolumn{3}{c}{64,27} & \multicolumn{3}{c}{69,33} & 2,99 \\
\hline
\end{tabular}

${ }^{1} \mathrm{Y}=58,666071+0,092024 \mathrm{x} \mathrm{R}^{2}=89,60 \% ;{ }^{2} \mathrm{Y}=77,52610+0,115357 \mathrm{x}+0,000431 \mathrm{x}^{2} \mathrm{R}^{2}=99,42 \% ;{ }^{3} \mathrm{Y}=87,919048+0,110595 \mathrm{x}+0,000971 \mathrm{x}^{2} \mathrm{R}^{2}=$ $99,24 \% ;{ }^{4} \mathrm{Y}=18,176786+0,062738 \mathrm{x} \mathrm{R}{ }^{2}=93,91 \% ;{ }^{5} \mathrm{Não}$ paramétrica; ${ }^{6} \mathrm{Y}=37,040536+0,225417 \mathrm{x} \mathrm{R}{ }^{2}=99,71 \% ;{ }^{7} \mathrm{Y}=59,997619+0,165476 \mathrm{x}-$ $0,001021 x^{2} R^{2}=98,87 \% ;{ }^{8} Y=59,288095+0,157143 x+0,000772 x^{2} R^{2}=98,73 \% ;{ }^{9} Y=63,307143+0,127143 x R^{2}=97,39 \% ; 10 Y=65,764286$ $+0,086190 \mathrm{x}^{2}=95,21 \%$; MG - média geral; CV - coeficiente de variação.

Segundo Grandis et al., (2018) as medidas biométricas apresentam alta correlação com peso e as medidas de carcaça dos animais, sendo este último considerado o elemento mais importante do animal, pois é nele que está contida a porção comestível de maior valor comercial. Gois et al. (2018) sugere que cordeiros devem ser abatidos com idade entre 90 e 100 dias, e peso vivo de 28 a $30 \mathrm{~kg}$, garantindo que haja maior proporção de músculos, menor proporção óssea e quantidade suficiente de gordura, preservando as propriedades de conservação e sensoriais da carcaça, parâmetros estes exigidos pelo mercado consumidor. Segundo experimento conduzido por Siqueira (2000), animais abatidos com $40 \mathrm{~kg}$ resultaram em carcaças com teores de gordura muito elevados, sendo menos apreciados pelos consumidores. Por outro lado, outros autores demonstram que o rendimento de carcaça em ovinos está entre 40 a $50 \%$ do peso vivo do animal (Osório et al., 1997; Alves et al., 2003). Sendo assim, o peso dos animais do presente estudo ( $>40 \mathrm{~kg}$ ), associado a idade avançada ( $>100$ dias) podem indicar que o produto final apresentaria maior rendimento de carcaça, porém com maior quantidade de gordura muscular, podendo não agradar o mercado consumidor.Oliveira et al. (2019) trabalharam com borregas em condições semelhantes ao do presente estudo recebendo ração extrusada na relação 30V:70C e encontraram uma espessura de gordura $1,75 \mathrm{~mm}$ em animais com escore de condição corporal de 3,34 e 8,10 cm² de área de olho de lombo e 40 kg. Já Pérez em et., (1998) abateram cordeiros da raça Santa Inês e Bergamácia com 53,43 
Aminoácidos protegidos na ração de borregas sobre o consumo, desempenho e comportamento ingestivo

$\mathrm{kg}$ e encontraram $2,74 \mathrm{~mm}$ de espessura de gordura na carcaça. Neste sentido, esperava-se que os animais do presente estudo apresentassem resultados semelhantes aos citados na literatura.

Houve efeito do período sobre o ECC, sendo que a partir dos 15 dias de experimento os animais tiveram aumento no ECC, estabilizando-se a partir do $60^{\circ}$ dia. Essas mudanças mais uma vez estão relacionadas com o início da puberdade dos animais, momento este em que há redução no CMS, e estabilização no desenvolvimento corporal, como observado anteriormente. O ECC manteve-se dentro do desejável para reprodução $(3,0$ a $4,0)$, e também para o abate $(2,5-3,5)$ para cordeiros Santa Inês e mestiços Dorper x Santa Inês (SOUSA et al., 2011). Tais resultados demonstram que tanto a utilização de aminoácidos protegidos, quanto o uso de fonte de proteína degradável no rúmen utilizada no tratamento controle foram capazes de manter os animais dentro do padrão de escore de condição corporal (ECC) indicado, gerando benefícios para o sistema de produção, principalmente no que se refere ao peso para abate, que está diretamente relacionado ao rendimento de carcaça. De acordo com Albuquerque et al., (2007) ovelhas com ECC acima ou igual a 2,5 apresentam melhor desempenho reprodutivo quando comparado com animais com ECC mais baixo. Sendo assim, ao final do experimento os animais encontravam-se aptos para entrarem na fase reprodutiva e para serem abatidos. Assim como no presente estudo, Zundt et al., (2003) também não observaram efeito de níveis de proteína no ECC de borregas.

O peso dos animais não foi influenciado pelos tratamentos e apresentou equação linear positiva durante o período avaliado. Podemos observar que mesmo havendo redução no CMS, o peso dos animais continuou aumentando. Observa-se que houve aumento de 35\% no peso vivo dos animais comparando o início do período experimental ao final. Este aumento foi o suficiente para resultar nos bons valores de ECC obtidos e estão relacionados ao efeito linear positivo encontrado para o comprimento corporal. Este fato está relacionado ao potencial genético dos animais, uma vez que o mesmo era constituído por um cruzamento entre duas raças especializadas para a produção de carne (Madruga et al., 2005). Sendo assim, mesmo atingindo a idade de puberdade o ganho de peso não foi afetado negativamente, ponto este importante para sistemas de produção animal destinados ao corte.

Houve efeito de interação entre os tratamentos e o período avaliado para as medidas de altura do posterior (AP) e comprimento corporal(CC), apresentando aumento linear durante o experimento. Resultados semelhantes foram encontrados por Costa Júnior et al., (2006), sendo de $67,6 \pm 6,0 \mathrm{~cm}$ e $68,1 \pm 4,9 \mathrm{~cm}$ respectivamente, para AP e CC, para borregas mantidas em sistemas de médio nível tecnológico. Este aumento pode estar relacionado com a idade dos animais, pois os mesmos encontravam-se em plena fase de desenvolvimento até o $60^{\circ}$ dia, momento este onde ocorre estabilização das medidas avaliadas. Assim como mencionado para altura do anterior (AA), largura de peito(LP) e ECC, esta estabilização explica a redução no CMS (Figura1).

O ganho médio diário (GMD) dos animais não foi influenciado pelos tratamentos e nem pelos períodos avaliados (Tabela 4). Os ganhos médios superaram as expectativas, visto que a dieta foi formulada para obtenção de ganhos de $200 \mathrm{~g}$ /animal/dia e todas as médias observadas apresentaram valores superiores a este.

Zundt et al. (2003) encontraram aumento linear no GMD para cordeiros e borregas de cinco meses e $30 \mathrm{~kg}$ em média recebendo níveis crescentes de proteína bruta na dieta, variando de 0,149 a 0,169 g/dia para fêmeas. Segundo este mesmo autor, o baixo ganho de peso pode ser atribuído à idade fisiológica dos animais, pois a capacidade máxima para esta variável ocorre até as 20 semanas de idade. No presente estudo, o ganho médio diário permaneceu 10,5\% acima do esperado fato este que contribuiu para os incrementos no peso vivo dos animais até a fase final do experimento, quando os mesmos se encontravam com aproximadamente oito meses de idade. Isso mostra que os animais foram mais eficientes, tendo em vista que o CMS ficou dentro do recomendado pela literatura (Tabela 2) e os ganhos foram superiores. Podemos atribuir estes resultados ao genótipo dos animais que eram provenientes do cruzamento de raças especializadas para a produção de carne e a qualidade nutricional das dietas experimentais.

A inclusão de aminoácidos encapsulados não influenciou $(\mathrm{p}<0,05)$ o comportamento ingestivo pelos animais (Tabela 5). O principal motivo para este resultado pode ser o fato das dietas não apresentarem diferenças nos teores de fibra, ou seja, as dietas continham a mesma relação V:C (Tabela 1). Araújo et al., (2019), trabalhando com borregas suplementadas com níveis crescentes de lisina e metionina protegidos da degradação ruminal em até $32 \mathrm{~g} /$ animal/dia também não observaram efeito no comportamento ingestivo dos animais. Segundo Van Soest (1994) o tempo dispendido para ruminação deve ser em torno de 8 horas por dia, podendo apresentar variações entre 4 a 9 horas, dependendo do teor de parede celular dos alimentos volumosos. No presente estudo os animais permaneceram em média 6 horas em ruminação, valor este dentro do recomendado na literatura e indicando bom funcionamento das funções ruminais.

O tempo dispendido em ócio pelos animais do presente estudo representou 57,12\% do total avaliado em 24 horas, enquanto para ingestão e ruminação este valor foi de $17,20 \%$ e 25,62\%, respectivamente. Araújo et al., (2019) encontrou valores de 69,66, 12,57 e 17,62\%, respectivamente para tempo gasto em ócio, ingestão e ruminação. 
Araújo, C. M. et al.

Tabela 4 - Ganho médio diário (GMD) em $\mathrm{g} / \mathrm{animal}$ /dia em função dos tratamentos, durante todo o período avaliado e em função dos períodos avaliados em dias e tratamentos.

\begin{tabular}{|c|c|c|c|c|c|}
\hline \multicolumn{3}{|c|}{ Tratamento } & \multicolumn{3}{|c|}{$0-90$ dias } \\
\hline & Controle & & & 226,22 & \\
\hline & Lis + Met & & & 217,22 & \\
\hline & MG & & & 221,72 & \\
\hline & $\mathrm{CV}$ & & & 18,03 & \\
\hline Tratamento & $0-15$ & $15-30$ & $0-30$ & $30-45$ & $0-45$ \\
\hline Controle & 274,00 & 201,99 & 231,00 & 247,33 & 230,88 \\
\hline Lis + Met & 258,33 & 196,00 & 217,75 & 277,33 & 230,22 \\
\hline MG & 266,16 & 198,99 & 224,37 & 262,33 & 230,55 \\
\hline $\mathrm{CV}$ & 24,28 & 33,06 & 19,36 & 35,45 & 17,60 \\
\hline Tratamento & $45-60$ & $0-60$ & $60-75$ & $0-75$ & $75-90$ \\
\hline Controle & 282,66 & 243,83 & 212,50 & 231,59 & 199,33 \\
\hline Lis + Met & 279,99 & 227,16 & 209,55 & 218,79 & 209,33 \\
\hline MG & 281,33 & 235,50 & 211,02 & 225,19 & 204,33 \\
\hline $\mathrm{CV}$ & 28,11 & 18,14 & 40,60 & 20,50 & 26,41 \\
\hline
\end{tabular}

MG - Média geral; CV - Coeficiente de variação.

Já o tempo gasto com mastigação apresentou média de 10 horas durante o período avaliado, sendo este a somatório do tempo despendido em ingestão e ruminação. Este processo de mastigação é fundamental para a manutenção da saúde ruminal, tendo em vista que é neste momento em que ocorre a maior produção de saliva que é a responsável pelo tamponamento do ambiente ruminal, evitando possíveis problemas, como a acidose (Van Soest, 1994).Estes resultando indicam a capacidade dos animais em ingerir, ruminar e mastigar o alimento. Sendo assim, a inclusão de aminoácidos encapsulados não prejudicou e nem melhorou a eficiências dos parâmetros de comportamento ingestivo dos animais.

Tabela 5- Efeito da inclusão de aminoácidos encapsulados sobre o comportamento ingestivo (minutos/dia) de borregas durante 24 horas.

\begin{tabular}{ccccc}
\hline Tratamento & ING & RUM & Ócio & MAST \\
\hline Controle & 264,00 & 360,33 & 815,66 & 624,33 \\
Lis + Met & 232,00 & 378,00 & 830,00 & 610,00 \\
\hline Período & ING & RUM & Ócio & MAST \\
\hline 1 & 259,75 & 354,50 & 825,75 & 614,25 \\
45 & 255,50 & 346,50 & 838,00 & 602,00 \\
90 & 228,75 & 406,50 & 804,75 & 635,25 \\
\hline MG & 248,00 & 369,16 & 822,83 & 617,16 \\
CV & 11,50 & 18,56 & 8,65 & 11,53 \\
\hline
\end{tabular}

Ing - ingestão; Rum - ruminação; Mast - mastigação; MG - média geral; CV - coeficiente de variação.

\section{Conclusão}

A inclusão de aminoácidos (lisina e metionina) encapsulados em até $4 \%$ na matéria seca do concentradonão altera o consumo de matéria, ganho de peso, desenvolvimento corporal e o comportamento ingestivo de borregas. 
Aminoácidos protegidos na ração de borregas sobre o consumo, desempenho e comportamento ingestivo

Aprovação do Comitê de Ética

O projeto foi aprovado pelo Comitê de ética em experimentação animal da Universidade Federal de Uberlândia, sob protocolo número 149/16.

\section{Referências}

Albuquerque F. H. M. A. R.; Martins G. A.; Rogério M. C. P.; Memória H. Q.; Sousa, R. T.; Simeão R. S. F.; Sales, C. M. P. V.; Magalhães A. F. B.; Macedo Júnior, G. L. 2007. Efeito da condição corporal antes da estação monta sobre o desempenho produtivo de ovelhas Santa Inês. Anais... XVII Congresso Brasileiro de Zootecnia, 29 a 31 maio, Londrina,PR. CD-ROM.

Alves, E. M.; Pedreira, M. S.; Oliveira, C. A. S.; Ferreira, D. N.; Moreira, B. S.; Freire, L. D. R.2010. Importância da sincronização do complexo proteína/energia na alimentação de ruminantes. Publicações em Medicina Veterinária e Zootecnia - PUBVET, 4. Disponível em: http://www. pubvet.com.br/artigo/2265/importacircncia-da-sincronizaccedilatildeodo-complexo-proteiacutenaenergia-na-alimentaccedilatildeo-deruminantes .

Alves, K.S.; Carvalho, F.F.R.; Ferreira, M.A.; Véras, A..S.C.; Medeiros, A.N.; Nascimento, J.F.; Nascimento, L.R.S.; Dos Anjos, A.V.A. 2003. Níveis de energia em dietas para ovinos Santa Inês: características de carcaça e constituintes corporais. Revista Brasileira de Zootecnia. 32: 1927 1936. Doi: http://dx.doi.org/10.1590/S1516-35982003000800017.

Araújo, C. M.; Macedo Junior, G. L.; Oliveira, K. A.; Varanis, L. F. M.; Assis, T. S.; Siqueira, M. T. S. 2019 Effect of the inclusion of lysine and methionine on the nutritional performance and ingestive behavior of lambs. Semina: Ciências Agrárias, 40: 957-970, Doi: 10.5433/1679-0359.2019v40n2p957 Disponível em: http:// www.uel.br/revistas/uel/index.php/semagrarias/article/view/33777.

Cezar, M. F.; Sousa, W. H. 2007. Carcaças ovinas e caprinas: obtenção, avaliação e classificação. Uberaba: Agropecuária Tropical, 232p.

Cochran, W.G. 1941The distribution of the largest of a set of estimated variances as a fraction of their total. Annals of Human Genetics, 11: 47-52. Doi: https://doi.org/10.1111/j.1469-1809.1941.tb02271.x.

Costa Júnior, G. S.; Campelo, J. E. G.; Azevêdo, D. M. M. R.; Martins Filho, R.; Cavalcante, R. R.; Lopes, J. B.; Oliveira, M. E. 2006. Caracterização morfométrica de ovinos da raça Santa Inês criados nas microrregiões de Teresina e Campo Maior, Piauí. Revista Brasileira de Zootecnia, 35: 2260-2267, Doi: http://dx.doi.org/10.1590/S151635982006000800009 .

Fischer, V.; Deswysen, A. G.; Dèspres, L.; Dutilleul, P.; Lobato, J. F. P. 1998. Padrões nectemerais do comportamento ingestivo de ovinos. Revista Brasileira de Zootecnia, 27: 362-369, 1998. Disponível em: https://www.academia.edu/34029978/Padr\%C3\%B5es_Nectemerais_ do_Comportamento_Ingestivo_de_Ovinos.

Gois, G. C.; Campos, F. S.; Pessoa, R. M. S.; Silva, A. A. F.; Ferreira, J. M. S.; Matias, A. G. S. 2018. Qualidade da carne de ovinos de diferentes pesos e condição sexual. PUBVET, 12: 1-9, 2018. Disponível em: https:// www.researchgate.net/publication/325209828_Qualidade_da_carne_ de_ovinos_de_diferentes_pesos_e_condicao_sexual.

Grandis, F. A.; Junior Fernandes, F.; Cunha, L. F. C.; Dias, C. B. A.; Ribeiro, E. L. A.; Constantino, C.2018. Relação entre medidasbiométricas e peso corporal emovinos da raça Texel.Veterinária e Zootecnia. 25: 1-8. Doi: https://doi.org/10.35172/rvz.2018.v25.57.

Kenyon, P. R., Maloney, S. K. e Blache, D. 2014. Review of sheep body condition score in relation to production characteristics. New Zealand Journal Agriculture, 57: 38- 64, Doi: https://doi.org/10.1080/0028 8233.2013.857698.
Kruskal, W. H; Wallis, W. A. Use of ranks in one-criterion variance analysis. Journal American StatisticalAssociation, 47: 583-621, 1952. Doi: $10.2307 / 2280779$. Disponível em: https://www.jstor.org/ stable/2280779?seq $=1$.

Macedo Junior, G. L., Magno, L. L., Oliveira, K. A., Araújo, C. M., Varanis, L. F. M., Assis, T. S. 2019. Uso de diferentes fontes de proteína e de gordura na composição de proteinados para ovinos: consumo e digestibilidade aparente. Veterinária Notícias, 25: 49-66. Doi: https:// doi.org/10.14393/VTN-v25n1-2019.4.

Madruga, M. S.; Sousa, W. H.; Rosales, M. D.; Cunha, M. G. G.; Ramos, J. L. F. 2005. Qualidade da carne de cordeiros Santa Inês terminados em diferentes dietas. Revista Brasileira de Zootecnia, 34: 309-315, 2005. Doi: http://dx.doi.org/10.1590/S1516-35982005000100035.

Monteiro, C. D.; Bicudo, S. D.; Toma, H. S. 2010. Puberdade em fêmeas ovinas. PUBVET, 4: Art. 856, 2010. Disponível em: http://www.pubvet. com.br/artigo/2313/puberdade-em-fecircmeas-ovinas.

NRC -National Research Council. 2007. Nutrient requirements of small ruminants. 362p.

Oliveira, K. A.; Macedo Junior, G. L.; Araújo, C. M.; Siqueira, M. T. S.; De Araújo, M. J. P.; Jesus, T .A. V. 2019. Productive parameters of growing lambs fed na extruded ration with diferente roughage: concentraterations. Semina: Ciências Agrárias, 40: 3641-3652. Doi: 10.5433/1679-0359.2019v40n6Supl3p3641. Disponível em: http:// www.uel.br/revistas/uel/index.php/semagrarias/article/view/35194 .

Osório, M. T.; Sierra, I.; Sañudo, C. 1997. Peso vivo ao abate, da carcaça e perdas por oreio, segundo a raça, sexo e idade em cordeiros. Anais da Reunião Anual da Sociedade Brasileira de Zootecnia, SBZ, Juiz de Fora, MG, Brasil, 34: 299-301.

Pérez, J.R.O.; Garcia, I.F.F.; Teixeira, J.C. 1998. Características de carcaça de cordeiros Santa Inês e Bergamácia com diferentes níveis de dejetos de suínos na dieta. Anais da Reunião Anual da Sociedade Brasileira de Zootecnia, SBZ, Botuicatu, SP, Brasil, 35: 299-301.

Rodrigues, R. C. 2010. Métodos de análises bromatológicas de alimentos: métodos físicos, químicos e bromatológicos. Pelotas: Embrapa Clima Temperado, 177p. Disponível em: https://www.infoteca.cnptia. embrapa.br/handle/doc/884390.

Shapiro, S. S.; Wilk, M. B. 1965. An Analysis of Variance Test for Normality, (Complete Samples). Biometrika, 52: 591-611. Doi: http:// dx.doi.org/10.2307/2333709

Siqueira, E. R. 2000. Sistemas de confinamento de ovinos para corte no Sudeste do Brasil. Anais do Simpósio Internacional Sobre Caprinos e Ovinos de Corte. Empresa Estadual de Pesquisa Agropecuária da Paraíba/AS, João Pessoa, PB, Brasil, 1:107-118.

Silva, D.J.; Queiroz, A.C. 2002. Análise de alimentos: Métodos Químicos e Biológicos. 3ª ed. Viçosa: Universidade Federal de Viçosa. 
Araújo, C. M. et al.

Souza, K. C.; Mexia, A. A.; Silva, S. C.; Garcia, J.; Silva Junior, L. S. 2011.Escore de condição corporal em ovinos visando a sua eficiência reprodutiva e produtiva. PUBVET, 5: Art. 997. Disponível em: http:// www.pubvet.com.br/artigo/1220/escore-de-condiccedilatildeocorporal-em-ovinos-visando-a-sua-eficiecircncia-reprodutiva-eprodutiva.

Stieven, I. C. B.; Rossi Junior, P.; Fernandes, S. R.; Zanetti, G. F.; Santana, M. H. A. 2011. Exigência e absorção de aminoácidos em bovinos. PUBVET, 7: Art. 1034. Disponível em: http://pubvet.com.br/ artigo/1495/exigecircncia-e-absorccedilatildeo-de-aminoaacutecidosem-bovinos.
Van Soest, P. J. 1994. Nutritional ecology of the ruminant. 2.ed.; Ithaca, New York: Cornell University, 476 p.

Zundt, M.; Macedo, F. A. F.; Martins, E. N.; Mexia, A. A.; Yamamoto, S. M. 2003. Desempenho de cordeiros alimentados com diferentes níveis proteicos. Revista Brasileira de Zootecnia, 3: 1307-1314. Doi: http:// dx.doi.org/10.1590/S1516-35982002000500030. 